\title{
Rotational acoustic resonances in cylindrical waveguides
}

\author{
Y. Duan \& M. McIver \\ Department of Mathematical Sciences, Loughborough University, Loughborough, \\ Leicestershire, LE11 3TU, UK
}

\begin{abstract}
An investigation is made into the existence of rotational acoustic resonances in a circular cylindrical waveguide, and their frequencies of oscillation are calculated numerically. The guide is assumed to contain a number of radial fins which have finite extent, and which are distributed at equal azimuthal angles around the guide. A variational principle is used to prove the existence of different types of localised, rotational motion, and the frequencies of these spinning modes are computed. The numerical method is based on the use of a Galerkin technique to solve the integral equation which arises in the solution of the governing Helmholtz equation. The variation of the spinning mode frequencies with the number of fins and type of mode is discussed, and a comparison with non-rotational resonances is made.
\end{abstract}

\section{Introduction}

Unforced fluid oscillations which have finite energy are known to occur in unbounded fluids and they are localised in regions in which there is some change in the properties or the geometry of the medium. In the context of water waves these oscillations are known as edge waves or trapped modes, whereas in acoustic waveguides they are known as acoustic resonances. Reviews of the 
types and occurrences of such oscillations are given by Parker \& Stoneman [19] and Evans \& Kuznetsov [8]. The practical consequences of the existence of these modes is that if the system is forced at a frequency near that of the mode, a large response of the fluid-structure system will occur. In particular Parker [18] made an experimental study of acoustic resonances in axial flow compressors and observe large vibrations of the blades. Clearly such large motions may result in damage to one or more components of the system and a general degradation in performance. Further work on the occurrence and effect of acoustic resonances in aeroengines has been done by Woodley \& Peake $[21,22]$ and Cooper \& Peake [4]

Mathematically these modes are eigenfunctions of a linear operator and the frequencies at which they occur are related to the eigenvalues of the operator. In general the modes which have been found and proven to exist have frequencies which are below some cut-off frequency in the problem, which means that they oscillate at frequencies at which no propagation of waves to infinity is possible. Examples of such modes are edge waves which propagate along periodic coastlines, which have been found by Evans \& Linton [9], and acoustic resonances in cascades of plates, which were found both experimentally and numerically by Parker $[16,17]$.

Proofs of the existence of trapped modes in two and three dimensions are given by Evans et al [7], Davies \& Parnovski [5] and Groves [11], and all of these proofs are based on variational principles. More recently the existence of edge waves, or as they are sometimes referred to in the electromagnetic literature Rayleigh-Bloch modes, has been established rigorously by Linton \& McIver [14]. Modes which have frequencies which are above a cut-off frequency in a guide have also been found numerically by McIver et al [15] and Evans \& Porter[10]. These modes are said to be embedded, as their frequencies correspond to eigenvalues which are embedded in the continuous spectrum of the relevant operator. Unfortunately this means that the usual variational 
arguments fail, and it is very difficult to prove rigorously that such modes exist.

The purpose of this work is to investigate the existence of trapped modes which correspond to rotational motion in a cylindrical acoustic waveguide which has circular cross-section. Such modes will be referred to as 'spinning modes' following the terminology of Parker [18], who found such modes experimentally in an axial flow compressor. Such a compressor contains rows of large numbers of blades arranged around a central core. A simple model of this is an infinitely long cylinder of circular cross-section, which contains a single row of thin, radial plates, distributed at equal angles about the centre of the cylinder. Linton \& McIver [13] compute 'standing' trapped modes for this geometry, but we will show that such modes are only one class of modes that exist, and additional spinning modes are possible.

The problem is formulated in the next section where matched eigenfunction expansions are used to generate an integral equation for the normal velocity on the matching plane, and then a Galerkin technique is used to solve the equation. A proof of the existence of spinning modes is given in $\S 3$ and numerical calculations of their frequencies are presented and discussed in $\S 4$.

\section{Formulation}

A circular, cylindrical waveguide contains $L$ radial fins which are uniformly distributed around the guide, as illustrated in figure 1. Cylindrical polar coordinates $(\rho, \theta, x)$ are used, where the $x$-axis points along the guide and the position of the fins is given by $\{\theta=m \beta, m=0, \ldots, L-1,0 \leq \rho \leq d,-a \leq x \leq a\}$,

where $\beta=2 \pi / L$. Trapped modes are functions $\operatorname{Re}\left[\phi e^{-i \omega t}\right]$, where $\phi$ is a nontrivial solution of the boundary-value problem

$$
\left(\nabla+k^{2}\right) \phi=0 \text { in the fluid }
$$




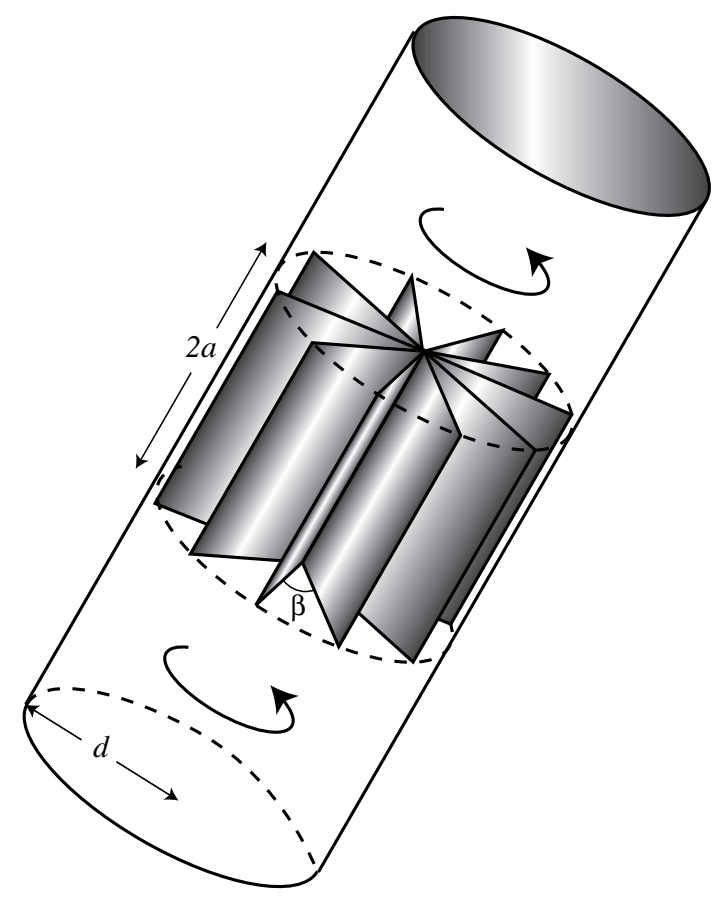

Fig. 1. Definition sketch

$$
\begin{gathered}
\frac{\partial \phi}{\partial \rho}=0 \text { on } \rho=d, \\
\frac{\partial \phi}{\partial \theta}=0 \text { on } \theta=m \beta, m=0, \ldots L-1,|x|<a \\
\phi \rightarrow 0 \text { as }|x| \rightarrow \infty
\end{gathered}
$$

The parameter $k=\omega / c$, where $\omega$ is the trapped mode frequency and $c$ is the speed of sound. An application of condition (4) to the eigenfunction expansion of $\phi$ in the far field ensures that the trapped mode has finite energy.

Linton \& McIver [13] restricted the type of modes which they considered to ones which satisfy $\phi=0$ on $\theta=m \beta,|x|>a, m=0, \ldots, L-1$. However in order to obtain modes which represent a rotational motion around the axis ('spinning modes') different conditions must be applied. To determine these, we first consider the scattering of the wave $J_{p}\left(j_{p, 1}^{\prime} \rho / d\right) e^{i p \theta+i\left(k^{2}-j_{p, 1}^{\prime 2} / d^{2}\right)^{1 / 2} x}$, where $J_{p}$ is the Bessel function of the first kind of order $p, p$ is a given integer and $j_{p, 1}^{\prime}$ is the first zero of $J_{p}^{\prime}$. As the geometry is unchanged after rotation through an angle $\theta=\beta$, the only change in the scattered wave field is due to the phase change of $e^{i p \beta}$ in the incident wave. Thus the scattered potential 
satisfies the pseudo-periodic condition

$$
\phi(\rho, \theta+m \beta, x)=e^{i p m \beta} \phi(\rho, \theta, x), \quad m=1, \ldots, L-1,0 \leq \theta<\beta, x>a .
$$

A spinning mode is sought which satisfies this same condition but whose frequency lies in the range $k d<j_{p, 1}^{\prime}$, which ensures that the potential decays down the guide. (This argument is similar to that used by Evans \& Linton [9] to obtain the condition for edge wave propagation along a line of plates in two dimensions.) Thus the potential may be solved for in the sector between one pair of fins and then extended to the whole domain with the use of (5). Furthermore the symmetry of the geometry about the plane $x=0$ allows the domain to be reduced to that part of the sector $\Omega=\{\rho, \theta, x: 0<\rho<d, 0<\theta<\beta, x>0\}$, by application of either the boundary condition for modes which are symmetric in $x$,

$$
\frac{\partial \phi}{\partial x}=0 \text { on } x=0,
$$

or that for modes which are antisymmetric in $x$

$$
\phi=0 \text { on } x=0
$$

In order to solve for $\phi$ in $\Omega$ the pseudo-periodic condition (5) is replaced by the so-called Bloch conditions

$$
\phi(\rho, \beta, x)=e^{i p \beta} \phi(\rho, 0, x), \quad \frac{\partial \phi}{\partial \theta}(\rho, \beta, x)=e^{i p \beta} \frac{\partial \phi}{\partial \theta}(\rho, 0, x), 0<\rho<d, x>a .
$$

We define region I to be $\{\rho, \theta, x: 0<\theta<\beta, 0<\rho<d, 0<x<a\}$ and region II to be $\{\rho, \theta, x: 0<\theta<\beta, 0<\rho<d, a<x<\infty\}$. An eigenfunction expansion is sought for $\phi$ in each region and the potential and normal derivative is matched on the common boundary. Details are given here for the potential which is symmetric in $x$.

The potential is written as

$$
\phi^{I}(\rho, \theta, x)=\sum_{m=0}^{\infty} \sum_{n=1}^{\infty} a_{m n} \frac{\cosh \alpha_{m n} x}{\alpha_{m n} \sinh \alpha_{m n} a} \psi_{m n}^{I}(\rho) \Psi_{m}^{I}(\theta)
$$


in region I and as

$$
\phi^{I I}(\rho, \theta, x)=\sum_{m=-\infty}^{\infty} \sum_{n=1}^{\infty} b_{m n} \frac{e^{-\beta_{m n}(x-a)}}{-\beta_{m n}} \psi_{m n}^{I I}(\rho) \Psi_{m}^{I I}(\theta),
$$

in region II, where

$$
\begin{gathered}
\psi_{m n}^{I}(\rho)=J_{m L / 2}\left(j_{m L / 2, n}^{\prime} \rho / d\right), \quad \Psi_{m}^{I}(\theta)=\cos (m L \theta / 2), \\
\psi_{m n}^{I I}(\rho)=J_{p+m L}\left(j_{p+m L, n}^{\prime} \rho / d\right), \quad \Psi_{m}^{I I}(\theta)=e^{i(p+m L) \theta}, \\
\alpha_{m n} d= \begin{cases}\left(j_{m L / 2, n}^{\prime 2}-(k d)^{2}\right)^{1 / 2}, & k d \leq j_{m L / 2, n}^{\prime}, \\
-i\left((k d)^{2}-j_{m L / 2, n}^{\prime 2}\right)^{1 / 2}, & k d>j_{m L / 2, n}^{\prime},\end{cases}
\end{gathered}
$$

and

$$
\beta_{m n} d=\left\{\begin{array}{l}
\left(j_{p+m L, n}^{\prime 2}-(k d)^{2}\right)^{1 / 2}, \quad k d \leq j_{p+m L, n}^{\prime} \\
-i\left((k d)^{2}-j_{p+m L, n}^{\prime 2}\right)^{1 / 2}, k d>j_{p+m L, n}^{\prime} .
\end{array}\right.
$$

(The term $\cosh \left(\alpha_{m n} x\right) / \sinh \left(\alpha_{m n} a\right)$ in (9) is replaced by $\sinh \left(\alpha_{m n} x\right) / \cosh \left(\alpha_{m n} a\right)$ in the potential which is antisymmetric in $x$.)

In order for $\phi$ to represent a spinning mode, $\phi \rightarrow 0$ as $x \rightarrow \infty$ and so from the expansion (10), for $b_{m n} \neq 0, \beta_{m n} d$ must be real and positive for all $m$ and $n$. From (14) this means that $p$ may be restricted to $p=0, \ldots, L-1$ and

$$
k d<\min \left(j_{p, 1}^{\prime}, j_{p-L, 1}^{\prime}\right) .
$$

In particular this means that there are no spinning modes when $p=0$. However if the requirement that $\phi$ be antisymmetric about the mid-plane of a sector is imposed, ie

$$
\phi(\rho, \beta / 2-\theta, x)=-\phi(\rho, \beta / 2+\theta, x), \quad 0<\theta<\beta / 2,
$$

then several of the coefficients in the expansions of $\phi$ in (9) and (10) must be set equal to zero. In particular $b_{0 n}=0$ and so a mode is possible with a frequency $k d<j_{L, 1}^{\prime}$ when $p=0$ and (16) is satisfied. This corresponds to one of the modes found by Linton \& McIver [13], and for simplicity this will simply 
Table 1

Cut-off frequencies for different $L$ and $p$

\begin{tabular}{|c|ccccccccccc|}
\hline$p \backslash L$ & 1 & 2 & 3 & 4 & 5 & 6 & 7 & 8 & 9 & 10 \\
\hline 0 & $j_{1,1}^{\prime}$ & $j_{2,1}^{\prime}$ & $j_{3,1}^{\prime}$ & $j_{4,1}^{\prime}$ & $j_{5,1}^{\prime}$ & $j_{6,1}^{\prime}$ & $j_{7,1}^{\prime}$ & $j_{8,1}^{\prime}$ & $j_{9,1}^{\prime}$ & $j_{10,1}^{\prime}$ \\
1 & & $j_{1,1}^{\prime}$ & $j_{1,1}^{\prime}$ & $j_{1,1}^{\prime}$ & $j_{1,1}^{\prime}$ & $j_{1,1}^{\prime}$ & $j_{1,1}^{\prime}$ & $j_{1,1}^{\prime}$ & $j_{1,1}^{\prime}$ & $j_{1,1}^{\prime}$ \\
2 & & & & $j_{2,1}^{\prime}$ & $j_{2,1}^{\prime}$ & $j_{2,1}^{\prime}$ & $j_{2,1}^{\prime}$ & $j_{2,1}^{\prime}$ & $j_{2,1}^{\prime}$ & $j_{2,1}^{\prime}$ \\
3 & & & & & & $j_{3,1}^{\prime}$ & $j_{3,1}^{\prime}$ & $j_{3,1}^{\prime}$ & $j_{3,1}^{\prime}$ & $j_{3,1}^{\prime}$ \\
4 & & & & & & & & $j_{4,1}^{\prime}$ & $j_{4,1}^{\prime}$ & $j_{4,1}^{\prime}$ \\
5 & & & & & & & & & & \\
\hline
\end{tabular}

be referred to as the $p=0$ mode. For general $p$ the symmetry of the geometry means that if $\phi(\rho, \theta, x)$ is a mode then so is $\phi(\rho,-\theta, x)$. From the expansion in (10) this means that the only other independent modes apart from that given by $p=0$ are given by $p=1, \ldots, L / 2$ for $L$ even and $p=1, \ldots,(L-1) / 2$ for $L$ odd. Thus (15) may be replaced by

$$
k d<j_{p, 1}^{\prime}, \quad p \neq 0 .
$$

Table 1 shows the number of possible modes for different numbers of fins and the cut-off frequencies below which $k d$ must lie.

An integral equation will now be derived and solved using a Galerkin procedure, which is based on the ideas of Porter \& Evans [20]. The analysis will be given for the case $p \neq 0$, as the case $p=0$ is covered by the work of Linton \& McIver [13].

Continuity of the potential and its normal derivative on $x=a$ gives

$$
\sum_{m=0}^{\infty} \sum_{n=1}^{\infty} a_{m n} \frac{\operatorname{coth}\left(\alpha_{m n} a\right)}{\alpha_{m n}} \psi_{m n}^{I}(\rho) \Psi_{m}^{I}(\theta)=\sum_{m=-\infty}^{\infty} \sum_{n=1}^{\infty} \frac{b_{m n}}{-\beta_{m n}} \psi_{m n}^{I I}(\rho) \Psi_{m}^{I I}(\theta),
$$


and

$$
f(\rho, \theta)=\sum_{m=0}^{\infty} \sum_{n=1}^{\infty} a_{m n} \psi_{m n}^{I}(\rho) \Psi_{m}^{I}(\theta)=\sum_{m=-\infty}^{\infty} \sum_{n=1}^{\infty} b_{m n} \psi_{m n}^{I I}(\rho) \Psi_{m}^{I I}(\theta)
$$

for $0<\rho<d$ and $0<\theta<\beta$ in both cases. Multiplication of (19) by $\rho \psi_{\mu \nu}^{I}(\rho) \Psi_{\mu}^{I}(\theta)$ and $\rho \psi_{\mu \nu}^{I I}(\rho) \overline{\Psi_{\mu}^{I I}(\theta)}, \mu=0,1, \ldots, \nu=1,2, \ldots$, in turn and integration over $D=\{\rho, \theta: 0<\rho<d, 0<\theta<\beta\}$, gives

$$
a_{\mu \nu} \frac{d^{2} \beta q_{\mu \nu}}{2 \varepsilon_{\mu}}=\int_{0}^{d} \int_{0}^{\beta} \rho f(\rho, \theta) \psi_{\mu \nu}^{I}(\rho) \Psi_{\mu}^{I}(\theta) d \rho d \theta
$$

and

$$
b_{\mu \nu} Q_{\mu \nu} \beta d^{2} / 2=\int_{0}^{d} \int_{0}^{\beta} \rho f(\rho, \theta) \psi_{\mu \nu}^{I I}(\rho) \overline{\Psi_{\mu}^{I I}(\theta)} d \rho d \theta
$$

respectively, where

$$
\begin{gathered}
\epsilon_{\mu}= \begin{cases}1, & \mu=0, \\
2, & \mu \neq 0,\end{cases} \\
q_{\mu \nu}= \begin{cases}\left(1-\frac{(\mu L / 2)^{2}}{j_{\mu L / 2, \nu}^{\prime 2}}\right)\left(J_{\mu L / 2}\left(j_{\mu L / 2, \nu}^{\prime}\right)\right)^{2}, & (\mu L / 2, \nu) \neq(0,1), \\
1, & (\mu L / 2, \nu)=(0,1)\end{cases}
\end{gathered}
$$

and

$$
Q_{\mu \nu}= \begin{cases}\left(1-\frac{(p+\mu L)^{2}}{j_{p+\mu L, \nu}^{\prime 2}}\right)\left(J_{p+\mu L}\left(j_{p+\mu L, \nu}^{\prime}\right)\right)^{2}, & (p+\mu L, \nu) \neq(0,1), \\ 1, & (p+\mu L, \nu)=(0,1) .\end{cases}
$$

The representations for $a_{\mu \nu}$ and $b_{\mu \nu}$ in terms of $f(\rho, \theta)$ will be substituted in (18) but first it is convenient to rearrange this equation so that any terms which correspond to imaginary $\alpha_{m n}$ are on the right-hand side of the equation and the remaining terms are on the left-hand side. (Terms in (18) for which $\alpha_{m n}$ is imaginary correspond to wave-like terms in $x$, in $\phi$ in region I.) The reason for this procedure is that it will result in a finite system of integral equations, which each have the same, positive-definite, Hermitian kernel. From (13) and (17) imaginary values of $\alpha_{m n}$ are given by the values of $m$ and $n$ for which

$$
j_{m L / 2, n}^{\prime}<k d<j_{p, 1}^{\prime}
$$


As $\max (p)=L / 2$ for $L$ even and $(L-1) / 2$ for $L$ odd, $p<m L / 2$ if $m \geq 1$ and so imaginary values of $\alpha_{m n}$ arise only when $m=0$. Suppose that there are $N$ such terms, that is $j_{0, N}^{\prime}<k d<j_{0, N+1}^{\prime}$, then (18) may be written as

$$
\begin{aligned}
& \sum_{n=N+1}^{\infty} a_{0 n} \frac{\operatorname{coth}\left(\alpha_{0 n} a\right)}{\alpha_{0 n}} \psi_{0 n}^{I}(\rho) \Psi_{0}^{I}(\theta)+\sum_{m=1}^{\infty} \sum_{n=1}^{\infty} a_{m n} \frac{\operatorname{coth}\left(\alpha_{m n} a\right)}{\alpha_{m n}} \psi_{m n}^{I}(\rho) \Psi_{m}^{I}(\theta) \\
& +\sum_{m=-\infty}^{\infty} \sum_{n=1}^{\infty} \frac{b_{m n}}{\beta_{m n}} \psi_{m n}^{I I}(\rho) \Psi_{m}^{I I}(\theta)=-\sum_{n=1}^{N} a_{0 n} \frac{\operatorname{coth}\left(\alpha_{0 n} a\right)}{\alpha_{0 n}} \psi_{0 n}^{I}(\rho) \Psi_{0}^{I}(\theta) .
\end{aligned}
$$

Substitution of (20) and (21) into (26) yields the integral equation for $f(\rho, \theta)$

$$
\int_{0}^{d} \int_{0}^{\beta} \rho^{\prime} f\left(\rho^{\prime}, \theta^{\prime}\right) K\left(\rho, \theta ; \rho^{\prime}, \theta^{\prime}\right) d \rho^{\prime} d \theta^{\prime}=\frac{\beta}{2} \sum_{n=1}^{N} \frac{a_{0 n} \cot \left(\alpha_{0 n}^{\prime} a\right) \psi_{0, n}^{I}(\rho) \Psi_{0}^{I}(\theta)}{\alpha_{0 n}^{\prime} d},
$$

where $\alpha_{0 n}^{\prime} d=-i \alpha_{0 n} d$ and

$$
\begin{aligned}
K\left(\rho, \theta ; \rho^{\prime}, \theta^{\prime}\right) & =\sum_{n=N+1}^{\infty} \frac{\operatorname{coth}\left(\alpha_{0 n} a\right)}{d^{2} \alpha_{0 n} d q_{0 n}} \psi_{0 n}^{I}(\rho) \Psi_{0}^{I}(\theta) \psi_{0 n}^{I}\left(\rho^{\prime}\right) \Psi_{0}^{I}\left(\theta^{\prime}\right) \\
& +\sum_{m=1}^{\infty} \sum_{n=1}^{\infty} \frac{2 \operatorname{coth}\left(\alpha_{m n} a\right)}{d^{2} \alpha_{m n} d q_{m n}} \psi_{m n}^{I}(\rho) \Psi_{m}^{I}(\theta) \psi_{m n}^{I}\left(\rho^{\prime}\right) \Psi_{m}^{I}\left(\theta^{\prime}\right) \\
& +\sum_{m=-\infty}^{\infty} \sum_{n=1}^{\infty} \frac{1}{d^{2} \beta_{m n} d Q_{m n}} \psi_{m n}^{I I}(\rho) \Psi_{m}^{I I}(\theta) \psi_{m n}^{I I}\left(\rho^{\prime}\right) \overline{\Psi_{m}^{I I}\left(\theta^{\prime}\right)}
\end{aligned}
$$

The function $f(\rho, \theta)$ is written as

$$
f(\rho, \theta)=\frac{\beta}{2} \sum_{n=1}^{N} \frac{a_{0 n} \cot \left(\alpha_{0 n}^{\prime} a\right)}{\alpha_{0 n}^{\prime} d} g^{(n)}(\rho, \theta)
$$

where $g^{(n)}(\rho, \theta)$ satisfies

$$
\mathcal{K} g^{(n)}(\rho, \theta)=\int_{0}^{d} \int_{0}^{\beta} \rho^{\prime} g^{(n)}\left(\rho^{\prime}, \theta^{\prime}\right) K\left(\rho, \theta ; \rho^{\prime}, \theta^{\prime}\right) d \rho^{\prime} d \theta^{\prime}=\psi_{0 n}^{I}(\rho) \Psi_{0}^{I}(\theta) .
$$

Each of the integral equations in (30) has the same kernel, and it follows immediately from (28) that

$$
\overline{K\left(\rho, \theta ; \rho^{\prime}, \theta^{\prime}\right)}=K\left(\rho^{\prime}, \theta^{\prime} ; \rho, \theta\right)
$$

and, for arbitrary $g(\rho, \theta)$ which is sufficiently smooth 


$$
\begin{aligned}
& \int_{0}^{d} \int_{0}^{\beta} \rho g(\rho, \theta)\left[\int_{0}^{d} \int_{0}^{\beta} \overline{K\left(\rho, \theta ; \rho^{\prime}, \theta^{\prime}\right) g\left(\rho^{\prime}, \theta^{\prime}\right)} \rho^{\prime} d \rho^{\prime} d \theta^{\prime}\right] d \rho d \theta \\
= & \sum_{n=N+1}^{\infty} \frac{\operatorname{coth}\left(\alpha_{0 n} a\right)}{\alpha_{0 n} d q_{0 n}}\left|\int_{0}^{d} \int_{0}^{\beta} \rho g(\rho, \theta) \psi_{0 n}^{I}(\rho) \Psi_{0}^{I}(\theta) d \rho d \theta\right|^{2} \\
+ & \sum_{m=1}^{\infty} \sum_{n=1}^{\infty} \frac{2 \operatorname{coth}\left(\alpha_{m n} a\right)}{\alpha_{m n} d q_{m n}}\left|\int_{0}^{d} \int_{0}^{\beta} \rho g(\rho, \theta) \psi_{m n}^{I}(\rho) \Psi_{m}^{I}(\theta) d \rho d \theta\right|^{2} \\
+ & \sum_{m=-\infty}^{\infty} \sum_{n=1}^{\infty} \frac{1}{\beta_{m n} d Q_{m n}}\left|\int_{0}^{d} \int_{0}^{\beta} \rho g(\rho, \theta) \psi_{m n}^{I I}(\rho) \overline{\Psi_{m}^{I I}(\theta)} d \rho d \theta\right|^{2} \geq 0
\end{aligned}
$$

provided that the infinite series converge, and is only equal to zero if $g=0$. Thus $\mathcal{K}$ is a positive definite, self-adjoint operator and so (30) may be solved for $g^{(n)}(\rho, \theta)$.

Multiplication of (29) by $\rho \psi_{0 \nu}^{I}(\rho) \Psi_{0}^{I}(\theta), \nu=1, \ldots, N$, integration over $D$ and substitution in (20) gives

$$
a_{0 \nu} d^{2} q_{0 \nu}=\sum_{n=1}^{N} a_{0 n} \frac{\cot \left(\alpha_{0 n}^{\prime} a\right)}{\alpha_{0 n}^{\prime} d} \int_{0}^{d} \int_{0}^{\beta} g^{(n)}(\rho, \theta) \psi_{0 \nu}^{I}(\rho) \Psi_{0}^{I}(\theta) \rho d \theta d \rho, \quad \nu=1, \ldots, N
$$

Thus, once the functions $g^{(n)}(\rho, \theta)$ are determined from (30), for a spinning mode to exist there must be a non-trivial solution of (33) for the variables $\left\{a_{0 \nu}\right\}, \nu=1, \ldots, N$. For this to be true, the determinant of the resulting system of equations must be zero and so, for a given number of fins $L$, each with length $a$, this last condition determines the frequency of the mode $k d$.

In order to solve the integral equation in (30) each function $g^{(n)}(\rho, \theta)$ is expanded in a complete set of functions over $D$. Clearly it would be possible to do this using a combination of Bessel functions in $\rho$ and trigonometric functions in $\theta$. However, in order to correctly model the singularity in the velocity at the ends of the plates at $\theta=0$ and $\theta=\beta$ it is more convenient to write an approximation to $g^{(n)}(\rho, \theta)$ in the form

$$
g^{(n)}(\rho, \theta)=\sum_{r=0}^{R} \sum_{s=1}^{S} A_{r s}^{(n)} g_{r s}(\rho, \theta)
$$


where

$$
g_{r s}(\rho, \theta)=\frac{i^{r}}{\pi} J_{r}\left(j_{r, s}^{\prime} \rho / d\right) \theta^{-1 / 2}(\beta-\theta)^{-1 / 2} T_{r}\left(\frac{2 \theta-\beta}{\beta}\right),
$$

and $T_{r}$ is a Chebyshev polynomial of the first kind. The only place where the behaviour of $g^{(n)}(\rho, \theta)$ may not be well-modelled is near $\rho=0$, where the form of the velocity is unknown. However, numerical experimentation suggests that the coefficients in the expansion for $g^{(n)}(\rho, \theta)$ in (34) decay faster than those in an expansion which uses trigonometric functions in $\theta$, and so (34) was used in all calculations presented in this work. Substitution of the expansion in (34) into (30), multiplication by $\overline{\rho g_{\mu \nu}(\rho, \theta)}$ and integration over $D$ yields, after considerable algebra,

$$
\begin{gathered}
\sum_{n=N+1}^{\infty} \frac{\operatorname{coth}\left(\alpha_{0 n} a\right)}{\alpha_{0 n} d q_{0 n}} A_{0 n}^{(l)} U_{00}^{n n} U_{0 \mu}^{n \nu} \overline{u_{0 \mu}}+ \\
\sum_{r=0}^{R} \sum_{s=1}^{S} A_{r s}^{(l)} \sum_{m=1}^{\infty} \sum_{n=1}^{\infty} \frac{2 \operatorname{coth}\left(\alpha_{m n} a\right)}{\alpha_{m n} d q_{m n}} U_{m r}^{n s} U_{m \mu}^{n \nu} u_{m r} \overline{u_{m \mu}}+ \\
\sum_{r=0}^{R} \sum_{s=1}^{S} A_{r s}^{(l)} \sum_{m=-\infty}^{\infty} \sum_{n=1}^{\infty} \frac{1}{\beta_{m n} d Q_{m n}} V_{m r}^{n s} V_{m \mu}^{n \nu} v_{m r} \overline{v_{m \mu}}=U_{0 \mu}^{l \nu} \overline{u_{0 \mu}} \\
l=1, \ldots, N, \quad \mu=0,1, \ldots, R, \nu=1,2, \ldots S,
\end{gathered}
$$

where

$$
\begin{array}{r}
U_{m r}^{n s}=\int_{0}^{1} \rho J_{r}\left(j_{r, s}^{\prime} \rho\right) J_{m L / 2}\left(j_{m L / 2, n}^{\prime} \rho\right) d \rho, \\
V_{m r}^{n s}=\int_{0}^{1} \rho J_{r}\left(j_{r, s}^{\prime} \rho\right) J_{p+m L}\left(j_{p+m L, n}^{\prime} \rho\right) d \rho, \\
u_{m r}=\int_{0}^{\beta} i^{r}(\pi)^{-1} \theta^{-1 / 2}(\beta-\theta)^{-1 / 2} T_{r}\left(\frac{2 \theta-\beta}{\beta}\right) \Psi_{m}^{I}(\theta) \\
=J_{r}(m L \beta / 4) \cos (m L \beta / 4+r \pi / 2) e^{i r \pi / 2},
\end{array}
$$

and

$$
\begin{aligned}
v_{m r} & =\int_{0}^{\beta} i^{r}(\pi)^{-1} \theta^{-1 / 2}(\beta-\theta)^{-1 / 2} T_{r}\left(\frac{2 \theta-\beta}{\beta}\right) \overline{\Psi_{m}^{I I}(\theta)} \\
& =J_{r}((p+m L) \beta / 2) e^{-i((p+m L) \beta / 2}
\end{aligned}
$$


Similarly, substitution of (35) into (33) gives

$$
a_{0 \nu} q_{0 \nu}-\sum_{n=1}^{N} a_{0 n} \frac{\cot \left(\alpha_{0 n}^{\prime} a\right)}{\alpha_{0 n}^{\prime} d} A_{0 \nu}^{(n)} U_{00}^{\nu \nu}=0, \quad \nu=1, \ldots, N
$$

The numerical solution of (36) and (41) will be discussed in $\S 4$ but first a proof of the existence of spinning modes will be given in the next section.

\section{Proof of the existence of spinning modes}

The existence of rotational acoustic resonances which are symmetric in $x$ will be established in this section for $p=0$ with the use of a standard variational argument. This extends the work of Davies \& Parnovski[5] and Groves[11] who proved that at least one type of mode may be supported by a thin plate in a guide, and it uses the ideas of Bonnet-Bendhia \& Starling[3], who studied Rayleigh-Bloch modes which are supported by electromagnetic diffraction gratings. As will be seen from the numerical results of the next section, modes which are antisymmetric in $x$ only exist when $a / d$ is above a critical value. A proof will be given for the existence of modes when $a / d>\pi / j_{p, 1}^{\prime}$, but from the numerical results this value of $a / d$ is not optimal and the authors were unable to determine what the critical value of $a / d$ should be.

The Sobolev space $H_{p L}^{1}(\Omega)$ is formed from the completion in the $H^{1}$ norm of the space of functions $C^{\infty}(\Omega)$ which have compact support and satisfy (8). A similar argument to that given by Bonnet-Bendhia \& Starling[3] shows that the operator $-d^{2} \nabla^{2}$, restricted to $H_{p L}^{1}(\Omega)$, has the essential spectrum

$$
\sigma_{\mathrm{ess}}=\left[\min \left\{j_{p, 1}^{\prime 2}, j_{p-L, 1}^{\prime 2}\right\}, \infty\right)=\left[j_{p, 1}^{\prime 2}, \infty\right)
$$

as the values of $p$ are restricted to $p=1, \ldots L / 2$ when $L$ is even and $p=$ $1, \ldots(L-1) / 2$ when $L$ is odd. (The essential spectrum for this operator may be thought of physically as the values of $(k d)^{2}$ for which waves that satisfy (8), 
may propagate in the empty guide.) A standard variational principle shows that the lowest point of the spectrum of the operator is given by

$$
\inf _{\psi \in H_{p L}^{1}(\Omega) \backslash\{0\}} Q[\psi], \quad \text { where } \quad Q[\psi]=\frac{d^{2} \int_{\Omega}|\nabla \psi|^{2} \mathrm{~d} \Omega}{\int_{\Omega}|\psi|^{2} \mathrm{~d} \Omega} .
$$

Thus if a function $\psi_{0} \in H_{p L}^{1}(\Omega) \backslash\{0\}$ is found for which $Q\left[\psi_{0}\right]<j_{p, 1}^{\prime 2}$ then there is an eigenvalue of the operator, $\left(k_{0} d\right)^{2}$, which is below the essential spectrum. This agrees with the intuitive ideas of the previous section which suggest that spinning modes may exist at frequencies for which waves cannot propagate down the guide.

The test function

$$
\psi_{0}= \begin{cases}J_{p}\left(j_{p, 1}^{\prime} \rho / d\right) \mathrm{e}^{i p \theta}+\epsilon^{1 / 2} i(1-x / a), & 0 \leq x \leq a, 0 \leq \theta \leq \beta, 0 \leq \rho \leq d, \\ J_{p}\left(j_{p, 1}^{\prime} \rho / d\right) \mathrm{e}^{i p \theta} \mathrm{e}^{-\epsilon(x / a-1)}, & x>a, 0 \leq \theta \leq \beta, 0 \leq \rho \leq d,\end{cases}
$$

where $\epsilon$ is a positive constant to be chosen, belongs to $H_{p L}^{1}(\Omega) /\{0\}$. Elementary integration gives

$$
\begin{gathered}
\int_{x=0}^{a} \int_{\theta=0}^{\beta} \int_{\rho=0}^{d}\left|\psi_{0}\right|^{2} \rho \mathrm{d} \rho \mathrm{d} \theta \mathrm{d} x=\beta a I_{1}+\frac{2 a}{p} \sin ^{2}\left(\frac{\beta p}{2}\right) I_{2} \epsilon^{1 / 2}+O(\epsilon), \\
\int_{x=a}^{\infty} \int_{\theta=0}^{\beta} \int_{\rho=0}^{d}\left|\psi_{0}\right|^{2} \rho \mathrm{d} \rho \mathrm{d} \theta \mathrm{d} x=\frac{\beta a}{2 \epsilon} I_{1}, \\
\int_{x=0}^{a} \int_{\theta=0}^{\beta} \int_{\rho=0}^{d}\left|\psi_{0}\right|^{2} \rho \mathrm{d} \rho \mathrm{d} \theta \mathrm{d} x=\beta a\left[\frac{j_{p, 1}^{\prime 2}}{d^{2}} I_{3}+p^{2} I_{4}\right]+O(\epsilon)
\end{gathered}
$$

and

$$
\int_{x=a}^{\infty} \int_{\theta=0}^{\beta} \int_{\rho=0}^{d} \nabla\left|\psi_{0}\right|^{2} \rho \mathrm{d} \rho \mathrm{d} \theta \mathrm{d} x=\frac{\beta a}{2 \epsilon}\left[\frac{j_{p, 1}^{\prime 2}}{d^{2}} I_{3}+p^{2} I_{4}\right]+O(\epsilon),
$$

where

$$
\begin{aligned}
I_{1} & =\int_{0}^{d} \rho J_{p}^{2}\left(j_{p, 1}^{\prime} \rho / d\right) \mathrm{d} \rho \\
I_{2} & =\int_{0}^{d} \rho J_{p}\left(j_{p, 1}^{\prime} \rho / d\right) \mathrm{d} \rho, \\
I_{3} & =\int_{0}^{d} \rho J_{p}^{\prime 2}\left(j_{p, 1}^{\prime} \rho / d\right) \mathrm{d} \rho,
\end{aligned}
$$

and

$$
I_{4}=\int_{0}^{d} \frac{1}{\rho} J_{p}^{2}\left(j_{p, 1}^{\prime} \rho / d\right) \mathrm{d} \rho
$$


The governing differential equation for $J_{p}$ is given in [1] 9.1 .1 by

$$
z^{2} J_{p}^{\prime \prime}+z J_{p}^{\prime}+\left(z^{2}-p^{2}\right) J_{p}=0
$$

Multiplication of (53) by $J_{p}(z) / z$ and integration from $z=0$ to $j_{p, 1}^{\prime}$ gives, after some manipulation

$$
\frac{j_{p, 1}^{\prime 2}}{d^{2}} I_{3}+p^{2} I_{4}=\frac{j_{p, 1}^{\prime 2}}{d^{2}} I_{1}
$$

Substitution of (45) - (52) and (54) into (43) shows that the Rayleigh quotient evaluated at $\psi=\psi_{0}$ is given by

$$
Q\left[\psi_{0}\right]=\mathrm{j}_{p, 1}^{\prime 2}\left[1-\frac{4 I_{2}}{\beta p I_{1}} \sin ^{2}\left(\frac{\beta p}{2}\right) \epsilon^{3 / 2}\right]+O\left(\epsilon^{2}\right) .
$$

From (49) $I_{1}$ is clearly positive and from (50) $I_{2}$ is also positive because $j_{p, 1}^{\prime}<$ $j_{p, 1}$, where $j_{p, 1}$ is the first positive zero of $J_{p}(z)$ and $J_{p}(z)>0$ for $0<z<j_{p, 1}$. Furthermore as $\beta p=2 \pi p / L$ and $p \leq L / 2, \sin (\beta p / 2)>0$. Thus $\epsilon$ may be chosen to be sufficiently small that

$$
Q\left[\psi_{0}\right]<j_{p, 1}^{\prime 2}
$$

and so there is an eigenvalue of the operator $-d^{2} \nabla^{2}$ restricted to $H_{p L}^{1}(\Omega)$, below the essential spectrum.

For modes which are antisymmetric in $x$ the test function

$$
\psi_{0}= \begin{cases}\sin \left(\frac{\pi x}{a}\right), & 0 \leq x \leq a, 0 \leq \theta \leq \beta, 0 \leq \rho \leq d, \\ 0, & x>a, 0 \leq \theta \leq \beta, 0 \leq \rho \leq d\end{cases}
$$

is used and a simple calculation shows that $Q\left[\psi_{0}\right]=\pi^{2} d^{2} / a^{2}$. This must be less than the cut-off $j_{p, 1}^{\prime 2}$ to guarantee that there is an eigenvalue below the essential spectrum, and so spinning modes which are antisymmetric in $x$ are guaranteed for

$$
\frac{a}{d}>\frac{\pi}{j_{p, 1}^{\prime}}
$$

However, as will be seen from the results in the next section, this is not a tight bound as there is numerical evidence that modes exist for values of $a / d$ below 
this.

\section{Results and discussion}

From the definition of $u_{m r}$ and $v_{m r}$ in (39) and (40) it is straightforward to show that $u_{m r} \overline{u_{m \mu}}, v_{m r} \overline{v_{m \mu}}$ and $\overline{u_{0, \mu}}$ must be real, and so the system of equations in (36) has real solutions $\left\{A_{r s}^{(i)}\right\}$. Thus the determinant of the system of equations for $\left\{a_{0 n}\right\}$ in (41), $\Delta$ is real. The frequencies of the spinning modes correspond to values of $k d$ at which $\Delta=0$, and these are computed as follows. The geometric parameters $L$ and $a / d$, and the mode number $p$ are fixed, and so for $p \neq 0$ any spinning mode frequencies are known to be in the range $0<k d<j_{p, 1}^{\prime}$. The coefficients $\left\{A_{r s}^{(l)}\right\}$ and the determinant $\Delta$ are calculated for a range of values of $k d$ in this interval, and smaller intervals in which $\Delta$ changes sign are located. To calculate $\left\{A_{r s}^{(l)}\right\}$ the indices $t=S r+s$ and $\tau=\mu S+\nu$ are introduced, so each pair $(r, s)$ and $(\mu, \nu)$ correspond to a unique value of $t$ and $\tau$ respectively, and then a NAG routine is used to solve the linear system of equations for the $S(R+1)$ unknowns $B_{t}^{(l)}=A_{r s}^{(i)}$. Once any intervals in which zeros of $\Delta$ lie are located approximately, a second NAG routine is used to determine the positions of these zeros more accurately and hence the value of $k d$ at which there is a spinning mode. The procedure is then repeated for different values of the parameters $L, a / d$ and $\mathrm{p}$.

Because the singularity at the ends of the plates is modelled accurately, numerical experimentation suggests that a very small matrix size, given by $R=2$ and $S=3$, is sufficient to obtain results which are accurate to three significant figures, provided that the infinite series in (36) are truncated at the larger values of 64 terms each. The main computational effort is contained in the numerical evaluation of $U_{m r}^{n s}, V_{m r}^{n s}$. An examination of the integrals in (37) and (38) shows that their integrands are highly oscillatory over at least part of the range of integration for large $m$ and $n$, and so a NAG routine which is 
tailored specifically for such integrals was used for their computation.

When there is one fin in the guide $(L=1)$ there is only one possible mode given by $p=0$, and when $L=2$ there are two independent modes given by $p=0$ and $p=1$. As has already been noted, the modes which correspond to $p=0$ are equivalent to those found by Linton \& McIver [13]. Those authors also found the mode for which $L=2$, and $p=1$. This mode spins with leading order azimuthal variation $e^{i \theta}$, and so there is an identical mode with azimuthal variation $e^{-i \theta}$ and a suitable combination of these two modes leads to a mode which is zero on the extensions of the fins and satisfies the condition imposed by Linton \& McIver [13]. Numerical calculations were checked against these results and good agreement obtained. In practice a rotational mode is more likely to be generated than a standing mode if there is some non-axisymmetric term in the forcing, such as a component of swirling flow.

When $L>2$ additional modes to those found by Linton \& McIver [13] may be obtained. Figure 2 illustrates the spinning mode frequencies for $L=3$ and these correspond to modes which are either symmetric or antisymmetric in $x$. It is clear that the mode which corresponds to $p=1$ has a frequency which is in the range $0<k d<j_{1,1}^{\prime}$, whereas the frequency of the mode which corresponds to $p=0$ lies in the range $j_{3 / 2,1}^{\prime}<k d<j_{3,1}^{\prime}$. As $a / d$ increases more and more modes appear, alternately symmetric and antisymmetric in $x$, and they all come in from the upper cut-off given in table 1 in $\S 2$ and, for $p \neq 0$ they tend to zero as $a / d \rightarrow \infty$. There is numerical evidence that whilst antisymmetric modes are not possible for all $a / d>0$, there are modes for plates of length $a / d<\pi / j_{1,1}^{\prime} \approx 1.7063$ when $p=1$. Thus the lower bound for $a / d$ for antisymmetric modes in (58) is not optimal.

When $p=0$ the lower bound for $k d$ corresponds to a cut-off below which there are no oscillatory modes in the inner region. Intuitively there must be at least one such mode for trapped motion to exist. From (9) and (13) there 


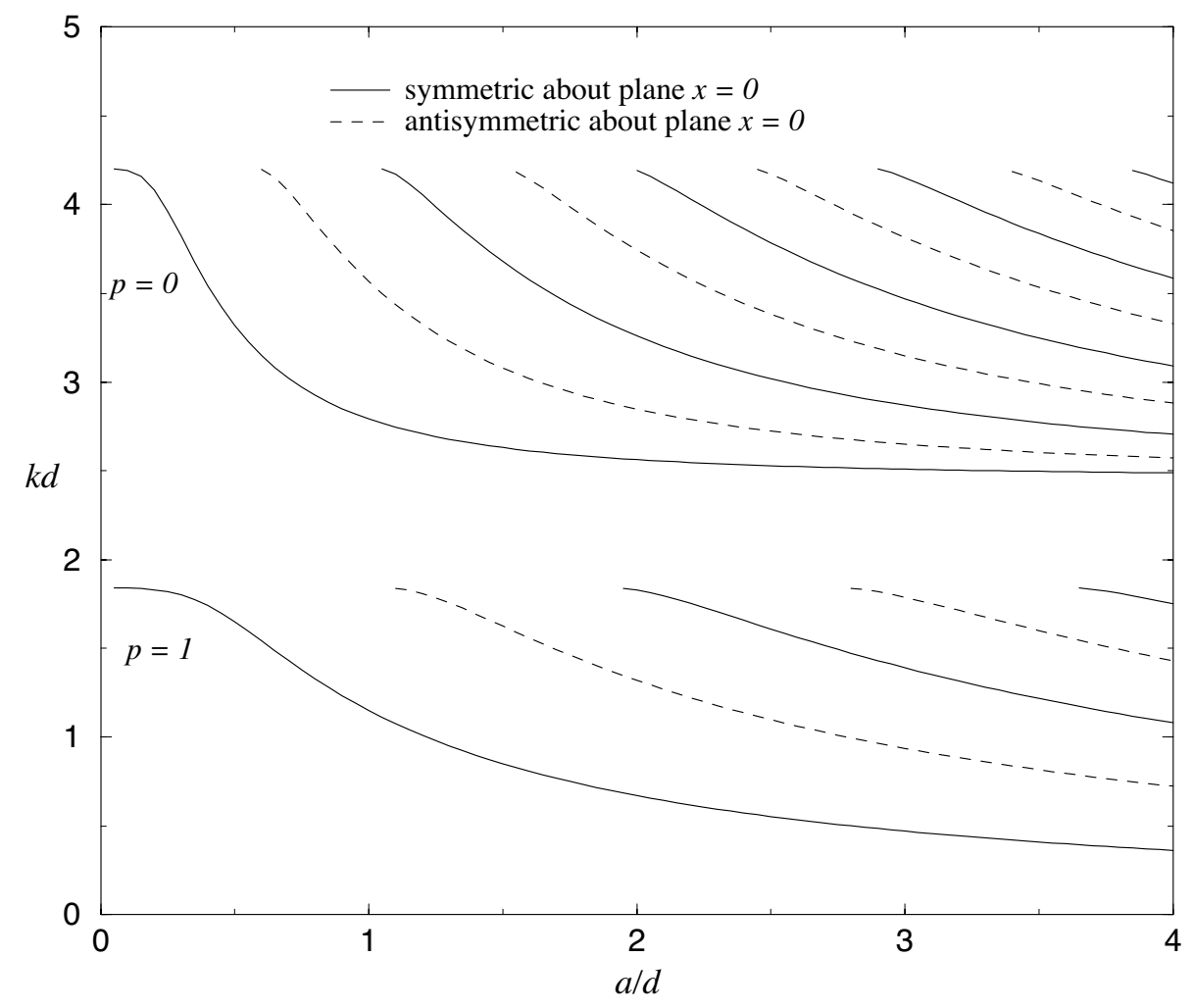

Fig. 2. Spinning modes for $L=3, p=0,1$.

is one oscillatory mode in the inner region if $k d<j_{1,1}^{\prime}$ but if the condition of antisymmetry about the centreline of the sector is imposed (which is required when $L$ is odd and $p=0$ ) then $a_{0 n}=0$ for all $n$ and so there are no oscillatory modes in the inner region when $k d<j_{3 / 2,1}^{\prime}$. This means that there is a gap $j_{1,1}^{\prime}<k d<j_{3 / 2,1}^{\prime}$ for which there are no spinning modes for $L=3$. This is an interesting feature which always occurs when $L$ is odd, and in general no trapped motion has been found for

$$
j_{(L-1) / 2,1}^{\prime}<k d<j_{L / 2,1}^{\prime}, \quad L \quad \text { odd }
$$

Thus if there is an external forcing with a frequency which corresponds to a value of $k d$ in this range, no large response of the system would be expected.

Figure 3 shows the spinning mode frequencies for $L=4$. The upper set of curves are for modes which correspond to $p=0$, and these are antisymmetric about the mid-plane of the sector. Their frequencies range from the cut-off for 


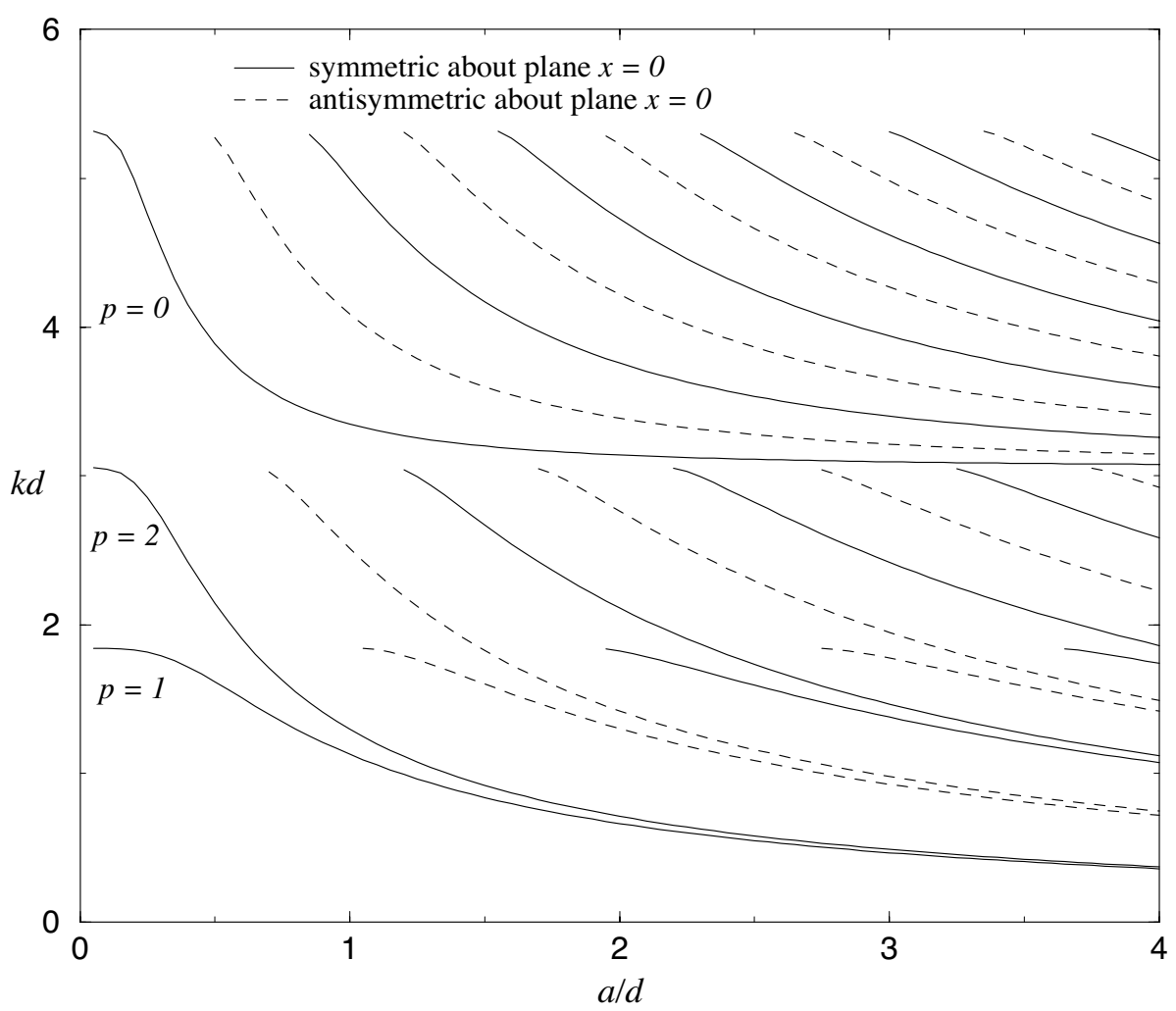

Fig. 3. Spinning modes for $L=4, p=0,1,2$.

the inner region $j_{2,1}^{\prime} \approx 3.0542$ to the cut-off for the outer region $j_{4,1}^{\prime} \approx 5.3176$. The middle set of curves are for $p=2$ and are confined to the region $0<k d<$ $j_{2,1}^{\prime} \approx 3.0542$, and on each curve the frequencies tend to zero as $a / d \rightarrow \infty$. The lowest set of curves represent modes which correspond to $p=1$, and their frequencies range from zero as $a / d \rightarrow \infty$, to the cut-off $j_{1,1}^{\prime} \approx 1.8412$. This last set of curves is exactly the same as for the case $L=2, p=1$ and, as noted by Linton \& McIver [13], this is unsurprising as a mode which is symmetric about the centreline of a sector formed by two fins is also a mode for four fins, and may be constructed from combinations of spinning modes. In this case both the potential and the normal derivative are continuous across one pair of the fins.

Each curve in the set for $p=2$ approaches a curve in the set $p=1$ as $a / d \rightarrow \infty$, and together they tend to zero. This behaviour may be explained by an examination of the side condition (41) as $a / d \rightarrow \infty$. If there is only 
one oscillatory term in the inner region this condition simplifies to give the transcendental equation for $k a$

$$
\frac{\cot (k a)}{k a}=\frac{2}{A_{01}} \frac{d}{a}
$$

Furthermore $\operatorname{coth}\left(\alpha_{m n} a\right) \rightarrow 1$ as $a / d \rightarrow \infty$, provided that $m$ and $n$ are not both zero, and so the system of equations for $A_{m n}$ in (36) tends to a system which is independent of $a / d$. Thus the right-hand side of (60) tends to zero as $a / d \rightarrow \infty$ and the limiting equation has solutions

$$
k d=\frac{(2 n-1) \pi d}{2 a}, \quad n \quad \text { integer }
$$

which are independent of the number of fins $L$ and the mode number $p$. To a first approximation the modes themselves may be shown to be given by

$$
\phi= \begin{cases}\cos \frac{(2 n-1) \pi x}{2 a}, & \text { in region } \mathrm{I}, \\ 0, & \text { in region II, }\end{cases}
$$

apart from in a transition region near $x=a$. Such a mode satisfies the Bloch conditions (8) for any values of $p$ and $\beta$.

A similar analysis shows that the asymptotic form of the antisymmetric frequencies is given by

$$
k d=\frac{n \pi d}{a}, \quad n \quad \text { integer }
$$

and the modes themselves are given by

$$
\phi= \begin{cases}\sin \frac{n \pi x}{a}, & \text { in region } \mathrm{I}, \\ 0, & \text { in region } \mathrm{II},\end{cases}
$$

Figure 4 compares the accurate calculations with the asymptotic frequencies for both the symmetric and antisymmetric modes when $L=4$ and $p=1$ or 2. For sufficiently large values of $a / d$ modes are possible for all permitted 


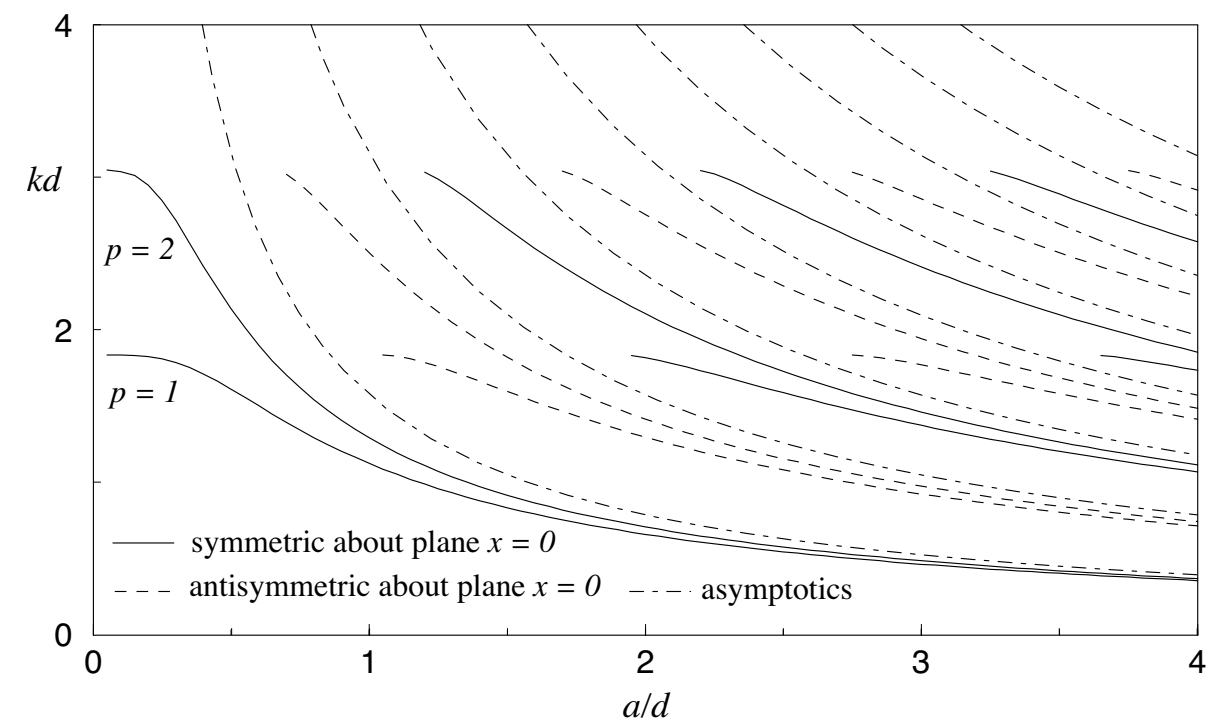

Fig. 4. Comparison of numerical and asymptotic results for $L=4, p=1,2$.

values of $p$ within a narrow range of $k d$, and the mode which is generated in practice will depend of the nature of any forcing. From the Bloch conditions in (8) spinning modes with the smallest value of $p$ correspond to those with the least oscillatory azimuthal variation and these are more likely to be generated than modes which correspond to larger values of $p$ (or $p=0$ ) unless the forcing is dominated by a particularly high frequency $\theta$ variation. In addition the frequencies of the modes for smaller values of $p$ are lower, and for values of $a / d \lesssim 1$ the differences may be quite significant. This becomes more noticeable when the number of fins is increased, as is shown in figure 5 where the four possible types of modes which exist when $L=7$ are presented.

Figure 5 illustrates clearly the gap in $k d$ in which there are no modes, namely

$$
j_{3,1}^{\prime}<k d<j_{3 / 2,1}^{\prime}
$$

but it also shows the same change in structure to the curves which was ob- 
served by Linton \& McIver [13] when $L=8$. The modes for the cases $p=0,1$ and 2 have similar behaviour to those for a smaller number of fins, but those for $p=3$ are different in character. For $a / d$ sufficiently large an extra set of modes exists and the two sets of curves appear to 'kiss' and then separate. Linton \& McIver [13] explained this phenomenon in terms of so-called diabolical points, which were first observed by Berry \& Wilkinson [2] in bounded regions. Physically the behaviour first occurs when two waves exist in the inner region, and so two different types of trapped oscillations are possible. From (25) this is when $j_{0,2}^{\prime}<k d<j_{3,1}^{\prime}$ (approximately $3.8317<k d<4.2012$ ). As $L$ increases more and more structure in the curves for the larger values of $p$ (and $p=0$ ) is expected, as the number of waves which exist in the inner region increases.

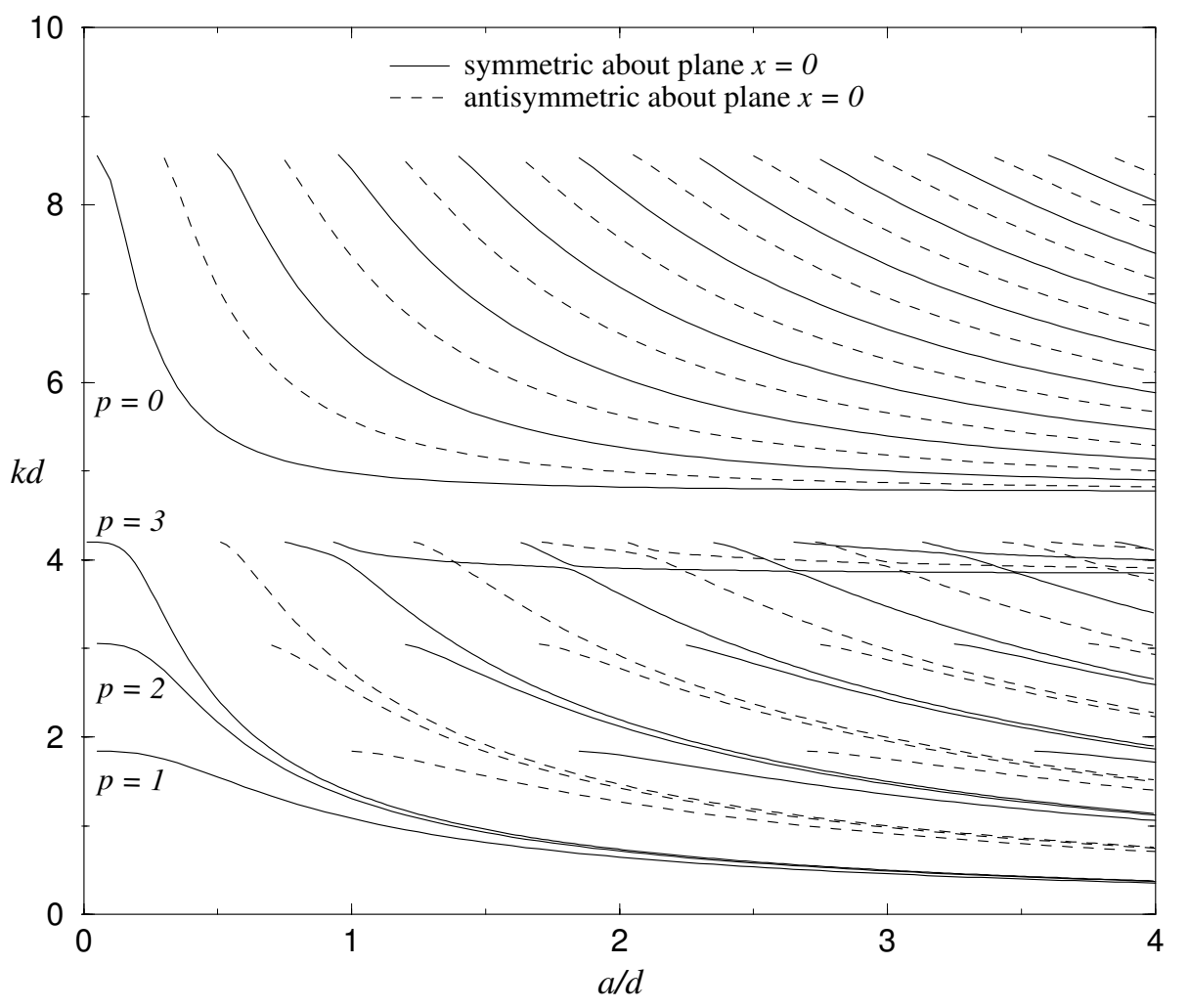

Fig. 5. Spinning modes for $L=7, p=0,1,2,3$. 


\section{Conclusion}

In this work the existence of spinning modes in a circular cylindrical guide which contains a number of radial fins, arranged at equal angles around the guide, has been investigated. Because of the symmetry of the geometry, the mathematical problem reduces to that of determining the eigenvalues of the Laplacian in a infinite cylindrical sector, with Neumann or Bloch conditions on the boundary of the sector. A proof of the existence of such modes was given and then a scheme based on a Galerkin method was used to locate the eigenvalues numerically. Graphs of the variation of spinning mode frequencies with plate length were presented for different mode numbers, and an investigation of the structure of these curves as the number of fins increased was made. For sufficiently large $a / d$ the behaviour of each curve was found to be independent of the number of fins and the mode number. However, the overall shape of each curve depends critically on the number of waves it is possible to trap in the inner region between the fins.

\section{References}

[1] Abramowitz, M. \& Stegun, I.A. 1965. Handbook of Mathematical Functions, Dover, New York.

[2] Berry, M.V, \& Wilkinson, M. 1985 Diabolical Points in the spectra of triangles. Proc. Roy. Soc. London. A392(1984) 15, 15-43

[3] Bonnet-Bendhia, A-S. And Starling, F. 1994. Guided waves by electromagnetic gratings and non-uniqueness examples for the diffraction problem. Mathematical Methods in the Applied Sciences. 17, 305-338.

[4] Cooper, A.J. \& Peake, N. 2000. Trapped acoustic modes in aeroengine intakes with swirling flow. J. Fluid Mechanics 419, 151-175. 
[5] Davies, E.B. \& Parnovski, L. 1998. Trapped modes in acoustic waveguides. Quarterly J. Mechanics Applied Mathematics 51,477-492.

[6] Evans, D. V., Linton, C. M. \& Ursell, F. 1993. Trapped mode frequencies embedded in the continuous spectrum Quarterly J. Mechanics Applied Mathematics 46, 253-274.

[7] Evans, D. V., Levitin, M. \& Vassiliev, D. 1994. Existence theorems for trapped modes. J. Fluid Mechanics 261, 21-31.

[8] Evans, D. V. And Kuznetsov, N. G. 1997. Trapped modes. In Gravity Waves on Water of Finite Depth,International Series on Advances in Fluid Mechanics, Computational Mechanics Publications, Southampton.

[9] Evans, D. V. And Linton, C. M. 1993. Edge waves along periodic coastlines. Quarterly J. Mechanics Applied Mathematics 46, 642-656.

[10] Evans, D. V. And Porter, R. 1998. Trapped modes embedded in the continuous spectrum. Quarterly J. Mechanics Applied Mathematics 52, 263-274.

[11] Groves, M.D. 1998. Examples of embedded eigenvalues for problems in acoustic waveguides Mathematical Methods in the Applied Sciences 21(6), 479488.

[12] Khallaf, N. S. A., Parnovski, L. \& Vassiliev, D. 2000. Trapped modes in a waveguide with a long obstacle. J. Fluid Mechanics 403, 251-261.

[13] Linton, C. M. \& McIver, P. 1998. Acoustic resonances in the presence of radial fins in circular cylindrical waveguides. Wave Motion 28, 99-117.

[14] Linton, C. M. \& McIver, M. 2002. The existence of Rayleigh-Bloch surface waves. J. Fluid Mech. 470, 85-90.

[15] McIver, M., Linton, C. M., McIver, P., Zhang, J. \& Porter, R. 2000. Embedded trapped modes for obstacles in two-dimensional waveguides. Quarterly J. Mechanics Applied Mathematics 54, 273-293.

[16] PARker, R. 1966. Resonance effects in wake shedding from parallel plates: some experimental observations. J. Sound Vibration 4, 62-72. 
[17] PARker, R. 1967. Resonance effects in wake shedding from parallel plates: calculation of resonance frequencies., J. Sound Vibration 5, 330-343.

[18] PARker, R. 1984. Acoustic resonances and blade vibration in axial flow compressors. J. Sound Vibration 92, 529-539.

[19] Parker, R. and Stoneman, S. A. T. 1989. The excitation and consequences of acoustic resonances in enclosed fluid flow around solid bodies. Proc. Instn Mech. Engrs 203, 9-19.

[20] Porter, R. And Evans, D.V. 1995. Complementary approximations to wave scattering by vertical barriers. J. Fluid Mechanics 294, 155-180.

[21] Woodley, B. M. \& Peake, N. 1999. Resonant acoustic frequencies of a tandem cascade. Part 1. Zero relative motion. J. Fluid Mechanics 393, 215-240.

[22] Woodley, B. M. \& Peake, N. 1999. Resonant acoustic frequencies of a tandem cascade. Part 2. Rotating blade rows. J. Fluid Mechanics 393, 241-256. 